\title{
Synergic Effect of Trimebutine Combined with Mosapride on Gastrointestinal Dysfunction and Visceral Pain Induced in Stress Models
}

\author{
Young-Joon Park ${ }^{1, a}$, Yong Sul Park ${ }^{2, a}$, Zoo Chul Chung ${ }^{2}$, Yun Sung Nam ${ }^{2}$, Yoon Hee Chung ${ }^{2,3}$, Kwan Hyung \\ Cho $^{1}$, Sung-Up Choi ${ }^{1, b}$, Uy Dong Sohn ${ }^{4}$, Eon Sub Park ${ }^{2,3}$, Hyun Dong Je ${ }^{5}$, Choongho Lee ${ }^{6}$, Moo Yeol Lee, \\ and Ji Hoon Jeong ${ }^{2,3, *}$
}

${ }^{1}$ Research Center, Samil Pharmaceutical Co. Ltd., Anyang 431-060,

${ }^{2}$ College of Medicine, Chung-Ang Universty, Seoul 156-756,

${ }^{3}$ Research Institute for Translational System Biomics, Chung-Ang Universty, Seoul 156-756,

${ }^{4}$ College of Pharmacy, Chung-Ang University, Seoul 156-756,

${ }^{5}$ Department of Pharmacology, College of Pharmacy, Catholic University of Daegu, Daegu 712-702, Republic of Korea

${ }^{6}$ Division of Gastroenterology and Hepatology, School of Medicine, Stanford University, Stanford, CA, USA

\begin{abstract}
The present study was undertaken to determine whether combined treatment with prokinetic trimebutine and mosapride has a synergic effect on gastrointestinal motility and visceral pain associated with gastrointestinal dysfunction. To develop effective gastroprokinetic agents with greater potencies than trimebutine or mosapride for the treatment of gastrointestinal tract disease, a mixture of trimebutine and mosapride was designed and prepared. In the present study, treatment with trimebutine alone showed a dose-dependent effect on propelling movements of normal small and large intestine in mice, whereas mosapride effected only small intestine motility. Co-administration of trimebutine with mosapride, a well-established prokinetic drug, produced a synergistic influence on normal small intestine motility, but demonstrated an unclear effect on large intestine motility, with a slight tendency to reduce the propelling time. In a stress model, the small and large intestine motilities were significantly decreased. The reduction of intestine motility was restored to a normal level and the restoring effect was more pronounced in the combined treatment with trimebutine plus mosapride than treatment with trimebutine or mosapride alone. Furthermore, treatment with trimebutine plus mosapride significantly decreased acute visceral pain which was not controlled by trimebutine or mosapride alone. These data suggest that combination therapy with trimebutine plus mosapride has a synergic effect on small and large intestine motility and visceral pain control in gastrointestinal disorders.
\end{abstract}

Key Words: Trimebutine, Mosapride, Gastrointestinal motility

\section{INTRODUCTION}

Gastrointestinal motility in mammals is regulated by complex and mutual interactions among neurotransmitters, hormones and spontaneous muscle contractility (Greenwood and Davison, 1987; Zenilman 1993). Therefore, decreased gastrointestinal motility may be involved in the etiology of indefinite gastrointestinal complaints such as poor appetite, abdominal distension, and vomiting associated with chronic gastritis, gastric neurosis, gastroptosis, or reflux esophagitis (Wingate et al., 2002). Even though there have been great advances in our understanding of the neurohumoral mechanisms controlling gastrointestinal motility in health and disease, the underlying pathogenesis of this condition is not fully understood, therefore, various drugs are used in the treatment of gastrointestinal motility disorders.

A gastroprokinetic agent is a type of drug which enhances gastrointestinal motility by increasing the frequency of con- www.biomolther.org

Open Access DOI: 10.4062/biomolther.2011.19.1.084

pISSN: 1976-9148 elSSN: 2005-4483

Copyright $\odot 2011$ The Korean Society of Applied Pharmacology
Received Aug 25, 2010 Revised Sep 16, 2010 Accepted Sep 18, 2010

\footnotetext{
*Corresponding Author

E-mail: jhjeong3@cau.ac.kr

Tel: +82-2-820-5688, Fax: +82-2-826-8752

'The authors contributed equally to this paper.

${ }^{b}$ Current affiliate: Korea Institute of Oriental Medicine, 483, Expo-ro, Yuseong-gu, Daejeon 305-811, Republic of Korea
} 
tractions in the small intestine or making them stronger, but without disrupting their rhythm (Reynolds and Putnam, 1992). They are used to treat a number of gastrointestinal disorders, including irritable bowel syndrome, gastritis, acid reflux disease, gastroparesis, and functional dyspepsia (Washabau, 2003; Chicella et al., 2005; Boyle et al., 2009). The promotility agents include drugs that act, at least in part, as 5-hydroxytryptamine-4 receptor agonists (Delco et al., 2007), the motilides, including erythromycin (Hawkyard and Koerner, 2007), a cholecystokinin receptor antagonist (Koop et al., 1986; Chua et al., 1994) and an opioid receptor antagonist that does not cross the blood brain barrier (Holzer, 2004).

Among a variety of prokinetic drugs, trimebutine is unique in being an enkephalin agonist which is clinically effective for the treatment of both hyperkinetic and hypokinetic motility disorders (Luttecke, 1978). The action of trimebutine in the gastrointestinal tract is mediated via an agonist effect on opiate receptors, the release of gastrointestinal peptides such as motilin, and modulation of the release of other peptides, including vasoactive intestinal peptide, gastrin, and glucagons (Delvaux and Wingate, 1997). As regards the mechanism of trimebutine's effect, several investigations indicated a direct action of trimebutine on the contraction of the isolated smooth muscles of the small intestine and colon. In these reports, trimebutine had both relaxing and excitatory effects on the smooth muscles (Nosaka et al., 1984; Takenaga et al., 1984a; Takenaga et al., 1984b; Takenaga et al., 1986). Shimada et al. (1990) reported that trimebutine has a $\mathrm{Ca}^{2+}$ antagonistic effect and inhibitory effect on the ACh-induced inward current, so it is expected to have a relaxing effect on the intestinal smooth muscle cells. Therefore, trimebutine may have biphasic effects on the intestinal smooth muscle cells.

Mosapride citrate is a novel benzamide gastrointestinal prokinetic agent whose structure contains a morpholine ring (Yoshida et al., 1993) and a selective 5-HT4 receptor agonist with no affinity for 5-HT1, 5-HT2, adrenaline $\alpha$ or dopamine D2 receptors (Sakurai-Yamashita et al., 1999; Kii et al., 2001). The principal metabolite of mosapride (M1) is a weak serotonin 5-HT3 antagonist. Mosapride has been known to be effective in improving overall symptoms in patients with gastrointestinal disorders, including chronic gastritis, gastrooesophageal reflux disease and functional dyspepsia (Curran et al., 2008).

In clinical practice, both trimebutine and mosapride, which has a different mechanism in exerting a prokinetic effect on GI motility, have been used individually in the treatment of patients with disturbances of gastrointestinal motility. Extensive work has been conducted to elucidate the beneficial and unfavorable effects of individual GI prokinetic agents in humans. However, little is known about the synergic effects of both agents in combination. In the current study, we investigated the prokinetic effect of trimebutine alone and the effect of trimebutine administered in combination with mosapride in a murine model. In addition, we examined if the combination treatment could attenuate acute visceral pain associated with gastrointestinal dysfunction.

\section{MATERIALS AND METHODS}

\section{Animals and treatment}

All animals were handled in accordance with Korean National Institute of Health guidelines for the humane care of laboratory animals. Five-week-old male ICR mice (Samtako, Korea) weighing $-20 \mathrm{~g}$ were used and allowed to acclimatize for 1 week before use in experiments. The animals were housed in an air-conditioned animal room maintained at 23-24 ${ }^{\circ} \mathrm{C}, 50-65 \%$ humidity, with a $12 \mathrm{~h}$ light-cycle. The animals had free access to food and water.

\section{Drugs}

For the model of small intestine motility, trimebutine, mosapride or trimebutine-mosapride complex (TM complex, $100: 5.29 \mathrm{w} / \mathrm{w}$ ) was dissolved in $25 \%$ ethanol. All drugs were administered by oral dosing, $30 \mathrm{~min}$ before each experiment.

\section{Gastrointestinal stress model}

Gastrointestinal stress was induced using the water-immersion restraint model (WIR). Efficacy of the stress model was confirmed by gastric mucosal lesion formation in mice. Prior to experiments, the animals were deprived of food, but not water for $12 \mathrm{~h}$. The animals were then placed in a restraint cage and immersed in the water bath $\left(22^{\circ} \mathrm{C}\right)$ to the level of the xiphoid process, as previously described (Takagi and Okabe, 1968). After $8 \mathrm{~h}$ stress, the animals were allowed to acclimatize for 1 $\mathrm{h}$ before use in experiments.

\section{Propelling test of small and large intestine}

All drugs were administered 30 min before the propelling test of the small or large intestine. For the small intestine propelling test (Zhang et al., 2005), conducted 30 min after test drug administration, $0.2 \mathrm{ml} / \mathrm{mouse}$ of $25 \% \mathrm{BaSO}_{4}$ suspended liquid was orally administered to the mice. All mice were sacrificed $20 \mathrm{~min}$ after the administration of $25 \% \mathrm{BaSO}_{4}$, and the entire small intestine was removed. The small intestine, from pylorus to the boundary of ileum and cecum, was isolated and its length was designated as "total length of small intestine". The length from pylorus to the foreland of $25 \% \mathrm{BaSO}_{4}$ suspension was designated as "BaSO ${ }_{4}$ suspension propelling length".

$$
\begin{aligned}
& \mathrm{BaSO}_{4} \text { suspension propelling ratio } \\
& =\frac{\mathrm{BaSO}_{4} \text { suspension propelling length }(\mathrm{cm})}{\text { total length of small intestine }(\mathrm{cm})] \times 100 \%}
\end{aligned}
$$

For the large intestine propelling test, conducted $30 \mathrm{~min}$ after test drug administration, $0.1 \mathrm{ml} / 10 \mathrm{~g}$ of $25 \% \mathrm{BaSO}_{4}$ suspended liquid was orally administered to the mice. After the $25 \% \mathrm{BaSO}_{4}$ suspension was excreted from the anus, the time interval between $25 \% \mathrm{BaSO}_{4}$ dosing and excretion was measured.

\section{Writhing test}

This test was selected as a model of acute peritoneovisceral pain, which can serve as a model of clinically relevant intestinal pain in humans (Dubinsky et al., 1987). Mice were injected i.p. with $10 \mathrm{ml} / \mathrm{kg}$ of $0.75 \%$ acetic acid (AA) and the number of writhes was counted during a 15 min period starting 5 min after administration of AA solution. A writhe was defined as a contraction of the abdominal muscles accompanied by an elongation of the body and extension of the hindlimbs. The drugs were administered p.o., 30 min before the AA injection.

\section{Statistical analysis}

All data were expressed as mean \pm SD. Statistical analy- 
sis was performed using the unpaired t test. Differences were considered significant when $p<0.05$.

\section{RESULTS}

\section{Effect of TM complex on $\mathrm{BaSO}_{4}$ suspension propelling ratio of small intestine in mice}

The $\mathrm{BaSO}_{4}$ suspension propelling ratio in the normal control group was $31.1 \pm 5.5 \%$. Compared with normal control, trimebutine $(25,50,100$ and $150 \mathrm{mg} / \mathrm{kg})$ induced increases of the $\mathrm{BaSO}_{4}$ suspension propelling ratio of the small intestine of $35.6 \pm 6.2 \%, 39.3 \pm 9.1 \%, 45.7 \pm 8.1 \%$ and $47.9 \pm 6.5 \%$, respectively (Fig. 1). Although different doses of trimebutine enhanced propelling movement with increasing dose, 100 and $150 \mathrm{mg} / \mathrm{kg}$ induced significant increases ( $p<0.05$ vs normal control). Therefore, we used trimebutine $(100 \mathrm{mg} / \mathrm{kg})$, mo-

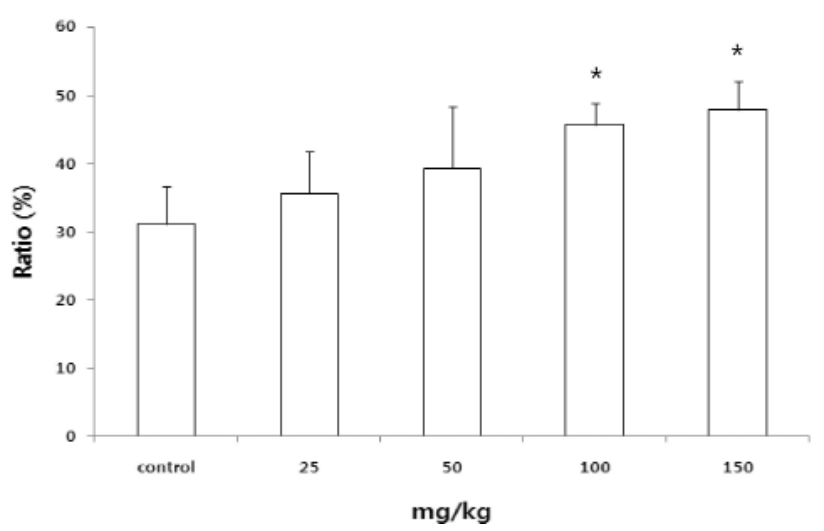

Fig. 1. Effect of trimebutine on $\mathrm{BaSO}_{4}$ suspension propelling ratio of small intestine in mice. Compared with normal control group, trimebutine $(100,150 \mathrm{mg} / \mathrm{kg})$ induced obvious increase of the propelling ratio of small intestine. Each value is the mean \pm S.E.M. of $5-8$ animals. ${ }^{*} p<0.05$ vs control.

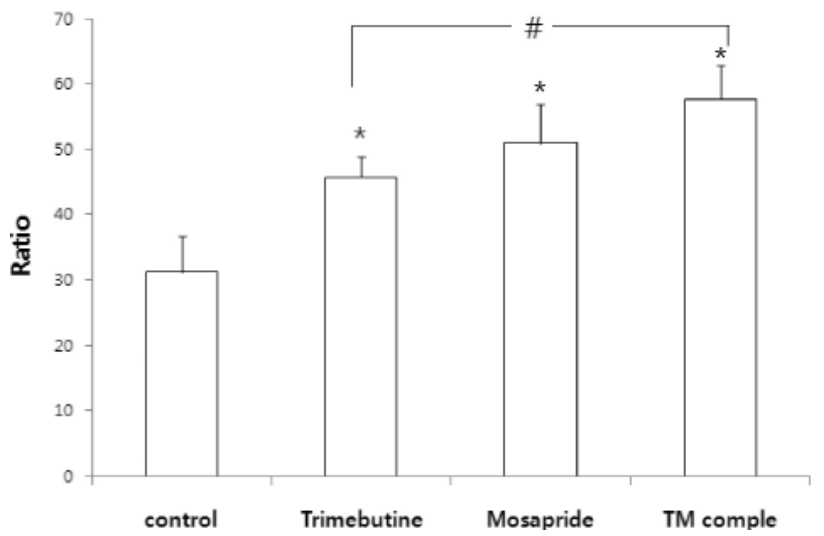

Fig. 2. Effect of $\mathrm{TM}$ complex on $\mathrm{BaSO}_{4}$ suspension propelling ratio of small intestine. Similar to trimebutine $(100 \mathrm{mg} / \mathrm{kg})$, mosapride $(5.29 \mathrm{mg} / \mathrm{kg}$ ) and TM complex (trimebutine $100 \mathrm{mg} / \mathrm{kg}$ and mosapride $5.29 \mathrm{mg} / \mathrm{kg}$ ) significantly increased the propelling ratio. TM complex was the most effective in $\mathrm{BaSO}_{4}$ suspension propelling movement of small intestine. Each value is the mean \pm S.E.M. of $5-8$ animals. ${ }^{*} p<0.05$ vs control, ${ }^{*} p<0.05$ vs trimebutine alone. sapride $(5.29 \mathrm{mg} / \mathrm{kg}$ ) and TM complex (trimebutine $100 \mathrm{mg} / \mathrm{kg}$ and mosapride $5.29 \mathrm{mg} / \mathrm{kg}$ ) in this study. Mosapride and TM complex significantly increased the propelling ratio $(p<0.05$ vs control), and reached $50.9 \pm 6.0 \%$ and $57.7 \pm 5.2 \%$, respectively (Fig. 2). WIR stress significantly decreased the BaSO4 suspension propelling ratio to $23.4 \pm 4.7 \%$ ( $p<0.05$ vs normal control) (Fig. 3). In the WIR stress model, trimebutine, mosapride and TM complex increased the propelling ratios to $30.8 \pm 4.5 \%, 28.4 \pm 5.1 \%$ and $40.8 \pm 4.8 \%$, respectively (Fig. 3). Trimebutine and TM complex significantly increased the propelling ratio ( $p<0.05$ vs normal control), and TM complex was more effective than trimebutine alone. Although TM complex was the most effective in stimulating $\mathrm{BaSO}_{4}$ suspension propelling movement of small intestine in both normal conditions and the WIR stress model, its effect was more pronounced in the WIR stress condition.

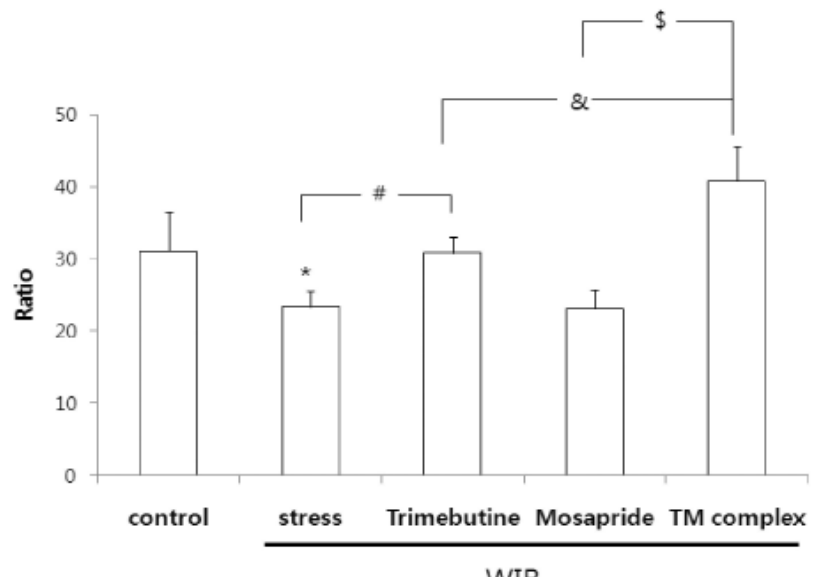

Fig. 3. Effect of $\mathrm{TM}$ complex on $\mathrm{BaSO}_{4}$ suspension propelling ratio of small intestine after WIR stress. WIR stress significantly decreased the propelling ratio. Trimebutine and TM complex significantly increased the propelling ratio, and TM complex was more effective than trimebutine alone. Each value is the mean \pm S.E.M. of 5-8 animals. ${ }^{*} p<0.05$ vs normal control, ${ }^{\#} p<0.05$ vs stress control, ${ }^{\&} p<0.05$ vs trimebutine, ${ }^{\$} p<0.01$ vs mosapride.

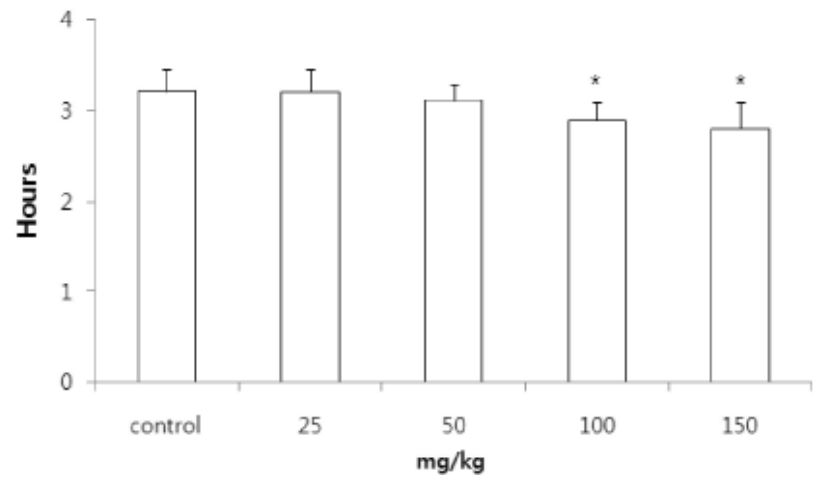

Fig. 4. Effect of trimebutine on $\mathrm{BaSO}_{4}$ suspension propelling time of large intestine. Compared with normal control, trimebutine (100 and $150 \mathrm{mg} / \mathrm{kg}$ ) induced significant decreases in the propelling time. Each value is the mean \pm S.E.M. of $5-8$ animals. ${ }^{*} p<0.05$ vs normal control. 


\section{Effect of TM complex on $\mathrm{BaSO}_{4}$ suspension propelling time of large intestine in mice}

The $\mathrm{BaSO}_{4}$ suspension propelling time of the large intestine in the normal control group was $3.18 \pm 0.24 \mathrm{~h}$. Compared with the normal control, trimebutine (100 and $150 \mathrm{mg} / \mathrm{kg}$ ) induced significant decreases $(p<0.05)$, and doses of $25,50,100$ and $150 \mathrm{mg} / \mathrm{kg}$ reached $3.21 \pm 0.25 \mathrm{~h}, 3.11 \pm 0.18 \mathrm{~h}, 2.86 \pm 0.24$ $\mathrm{h}$ and $2.81 \pm 0.29 \mathrm{~h}$, respectively (Fig. 4). TM complex (trimebutine $100 \mathrm{mg} / \mathrm{kg}$ plus mosapride $5.29 \mathrm{mg} / \mathrm{kg}$ ) significantly decreased the propelling time to $2.49 \pm 0.30 \mathrm{~h}(p<0.05$ vs normal control) (Fig. 5). WIR stress significantly increased the $\mathrm{BaSO}_{4}$ suspension propelling time to $4.15 \pm 0.28 \mathrm{~h}(p<0.05$ vs normal control) (Fig. 6). In stress model group, trimebutine, mosapride and TM complex induced significant decreases in the propelling time ( $p<0.05$ vs stress control), and reached 3.63 $\pm 0.25 \mathrm{~h}, 3.54 \pm 0.37 \mathrm{~h}$ and $2.87 \pm 0.25 \mathrm{~h}$, respectively (Fig. $6)$. The TM complex was more effective in modulating large

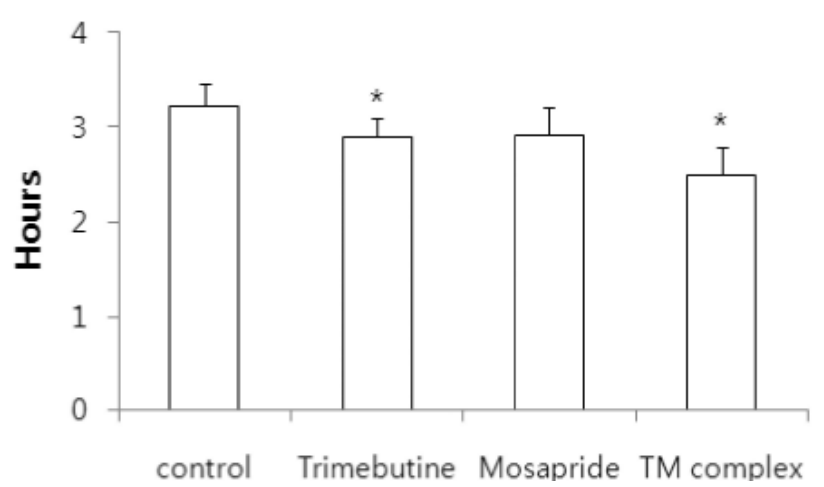

Fig. 5. Effect of $\mathrm{TM}$ complex on $\mathrm{BaSO}_{4}$ suspension propelling time of large intestine. TM complex (trimebutine $100 \mathrm{mg} / \mathrm{kg}$ and mosapride $5.29 \mathrm{mg} / \mathrm{kg}$ ) significantly decreased the propelling time. Each value is the mean \pm S.E.M. of $5-8$ animals. ${ }^{*} p<0.05$ vs normal control.

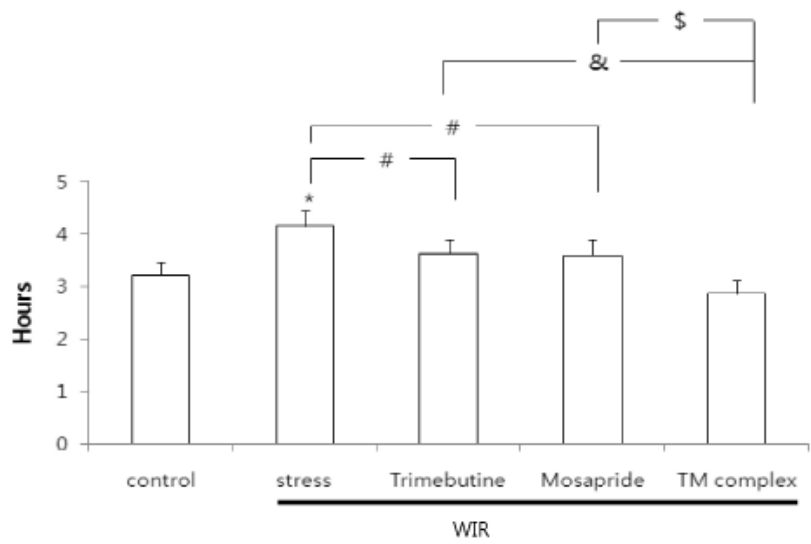

Fig. 6. Effect of $\mathrm{TM}$ complex on $\mathrm{BaSO}_{4}$ suspension propelling time of large intestine after WIR stress. WIR stress significantly increased the propelling time. Trimebutine, mosapride and TM complex induced significant decreases in the propelling time. Among them, TM complex was the most effective in modulating the propelling movement of large intestine. Each value is the mean \pm S.E.M. of $5-8$ animals. ${ }^{*} p<0.05$ vs normal contro, ${ }^{*} p<0.05$ vs stress control, ${ }^{\&} p<0.05$ vs trimebutine, ${ }^{\$} p<0.01$ vs mosapride. intestine $\mathrm{BaSO}_{4}$ suspension propelling movement in the WIR stress model than in normal conditions.

\section{Antinociceptive effect of TM complex on writhing behav- iors in mice}

Acetic acid administration produced a typical pattern of writhing behavior. Time-response curves obtained for trimebutine $(100 \mathrm{mg} / \mathrm{kg})$, mosapride $(5.29 \mathrm{mg} / \mathrm{kg})$ and TM complex (trimebutine $100 \mathrm{mg} / \mathrm{kg}$ and mosapride $5.29 \mathrm{mg} / \mathrm{kg}$ ) are depicted in Fig. 7. The abdominal contraction number for the control group was comparably increased at 20 min after AA administration, and remained elevated at $60 \mathrm{~min}$. The numbers of writhes in drug-treated groups were increased at $30 \mathrm{~min}$ after AA administration, and began to decrease at $40 \mathrm{~min}$. Thus,

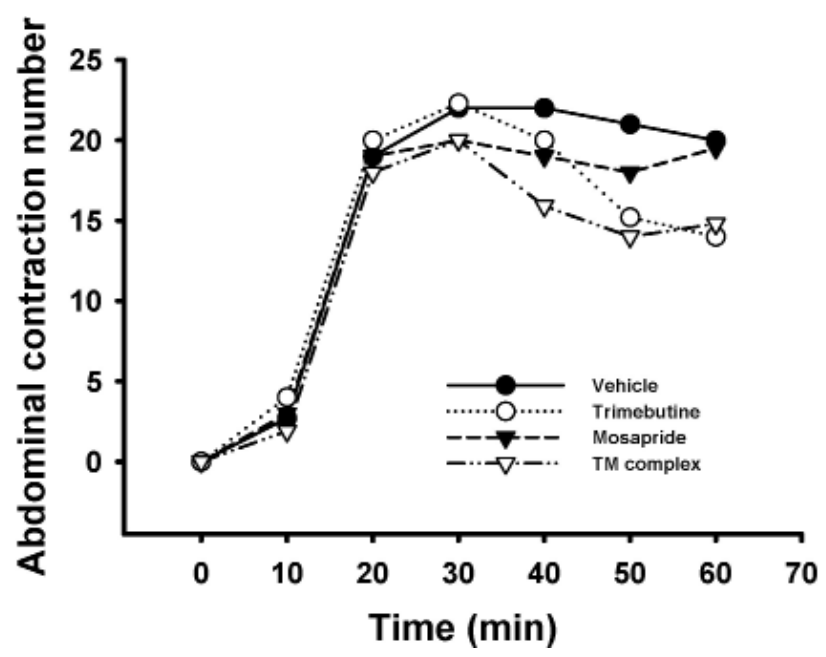

Fig. 7. Time-dependent effect of TM complex on writhing behavior. At 40 min after AA injection, trimebutine, mosapride and TM complex induced significant reductions in the number of writhes, compared with the control group. Each value is the mean \pm S.E.M. of 5-8 animals.

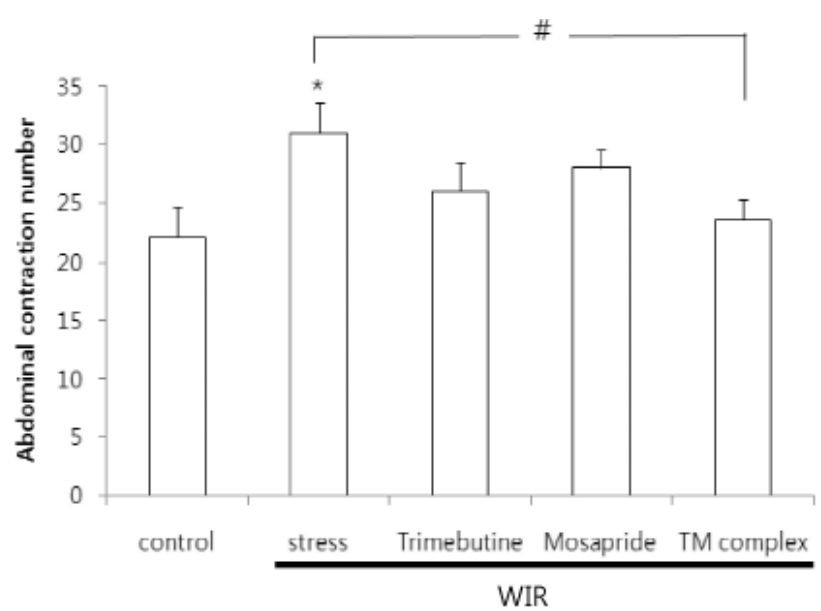

Fig. 8. Effect of TM complex on writhing behavior after WIR stress. In stress model group, TM complex was the most effective in decreasing the number of writhes. Each value is the mean \pm S.E.M. of $5-8$ animals. ${ }^{*} p<0.05$ vs normal control, ${ }^{*} p<0.05$ vs stress control. 
the number of writhes was counted during a 15 min period starting 40 min after AA injection. In the mouse writhing test, the number of writhes in the normal control group was 22.2 \pm 3.5 . The number of writhes in the trimebutine, mosapride and TM complex-treated groups were $20.1 \pm 3.2$, $18.2 \pm 3.4$ and $15.8 \pm 4.6$, respectively (Fig. 8). Trimebutine, mosapride and TM complex induced significant reductions in the number of writhes, compared with the control group $(p<0.05)$. WIR stress significantly increased the abdominal contraction number ( $p<0.05$ vs normal control) (Fig. 8). In stress model group, trimebutine and TM complex induced significant decreases in the number of writhes (trimebutine, $p<0.05$ vs stress control; TM complex $p<0.01$ vs stress control). TM complex was the most effective regimen for reducing the writhing number in the WIR stress model, suggesting that, in stressed conditions,

TM complex may have a greater antinociceptive effect than trimebutine or mosapride alone.

\section{DISCUSSION}

In this study, trimebutine alone showed a dose-dependent effect on propelling movement of normal small and large intestine in mice, whereas mosapride had an effect only on small intestine motility. Co-administration of trimebutine with mosapride, a well-established prokinetic drug, produced a synergistic influence on normal small intestine motility, but showed an unclear effect on large intestine motility with a slight tendency to reduce the propelling time. In a stress model, both the small and large intestine motilities were significantly decreased. The reduced intestine motilities were restored to normal levels and the restorative effect was more pronounced following the combined treatment with trimebutine plus mosapride than with trimebutine or mosapride alone. Furthermore, the trimebutine plus mosapride treatment significantly decreased acute visceral pain, which was not controlled by trimebutine or mosapride alone. These data suggest that combination therapy with trimebutine and mosapride produces a synergic effect on small and large intestine motility and visceral pain control in gastrointestinal disorders.

Trimebutine is a unique prokinetic agent that it is an enkephalin agonist clinically effective for the treatment of both hyperkinetic and hypokinetic motility disorders (Luttecke, 1978). Especially, trimebutine has a dual effect, showing a stimulatory effect on the hypomotile gastrointestinal tract, but an inhibitory effect on the hypermotile tract. In the present study, trimebutine has produced an increase in motility in normal small and large intestine. The acceleration of motor activity with trimebutine was more evident in the hypo-motility condition in small and large intestine induced by WIR stress, suggesting that trimebutine may be more effective in conditions of gastrointestinal disorder. The action of trimebutine in the gastrointestinal tract may be due to various pharmacological mechanisms mediated via an agonist effect on peripheral mu, kappa and delta opiate receptors, the release of gastrointestinal peptides such as motilin, and modulation of the release of other peptides, including vasoactive intestinal peptide, gastrin, and glucagons (Tanaka, 1998; Uchiyama et al., 2000; Kountouras et al., 2002). In clinical practice, trimebutine has been used in the treatment of patients with disturbances of gastrointestinal motility, such as abdominal pain, dyspepsia and irritable bowel syndrome (Delvaux et al., 1997).
Mosapride is also a prokinetic agent that acts selectively on the 5- $\mathrm{HT}_{4}$ receptors (Kii et al., 2001; Curran et al., 2008). It has been reported that the prokinetic effect of mosapride on gastrointestinal motility was somewhat different from that of other $5-\mathrm{HT}_{4}$-receptor agonists such as cisapride, renzapride and zacopride (Yoshida et al., 1991). Specifically, mosapride selectively enhanced motility in the upper gastrointestinal tract, such as in the stomach and intestine, whereas cisapride and zacopride stimulated the motility in all sites of the gastrointestinal tract from the stomach and the colon in conscious dogs (Mine et al., 1997). The previous results are in agreement with the present data showing that mosapride alone induced a more pronounced increase of motility in normal small intestine than that in normal large intestine. However, the prokinetic action of mosapride was evident in a hypo-motile condition induced by WIR stress in both small and large intestine. The present data show that mosapride is able to normalize the pathological state in both the upper and lower intestine.

In clinical practice, combination therapy with pharmacologically different drugs has sometimes produced a synergic effect by exerting a unique pharmacological action. The present data from animal experiments demonstrated that TM complex increased the motility of small intestine and large intestine more than trimebutine or mosapride alone. Under normal conditions, there was a significant motility increase with TM complex compared to trimebutine alone, whereas there was no significant change of small intestine propelling effect between mosapride alone and TM complex. This non-significant effect between mosapride alone and TM complex might be due to the unique action of mosapride, selectively enhancing the motility of the upper gastrointestinal tract. This means there is no synergic effect under normal conditions. In contrast, the synergic effect occurred in hypo-motility conditions in the stress model. It has been suggested that in irritable bowel syndrome or other functional disorders. trimebutine has a favorable effect that enhanced upper intestine motility (Kountouras et al., 2002). In contrast, trimebutine has been reported to have an inhibitory effect on a hypermotile lower intestinal tract (Matsue et al., 1990). These effects are believed to result from a direct action on the intestinal smooth muscle. The potentiation of trimebutine by mosapride might result from different pharmacologic mechanisms. The ability of mosapride in enhancing the motility of the upper gastrointestinal tract adds to the modulatory effect of trimebutine on gastrointestinal motility, since mosapride selectively acts on the $5-\mathrm{HT}_{4}$ receptor, which mainly exists in the intestine (Curran et al., 2008). It stimulates antroduodenal motility and accelerates intestinal transit in mice with low gastrointestinal motility.

Besides its regulatory effects on gastrointestinal motility through its weak opioid properties, trimebutine is also able to influence the activity of visceral afferents, which could be due to its blocking effect on sodium channels (Roman et al., 1999). Therefore, trimebutine has shown its beneficial effects on abdominal pain in patients with gastrointestinal dysfunction. Mosapride also alleviated abdominal pain and abdominal distension, indicating that mosapride is useful for the treatment of irritable bowel syndrome patients with constipationtype irritable bowel syndrome (Odaka et al., 2006). Although trimebutine or mosapride alone did not attenuate visceral pain in a stress model, the co-treatment with trimebutine plus mosapride significantly decreased acute visceral pain. However, despite increasing insights into the exact mechanisms under- 
lying the direct actions of trimebutine and mosapride, it remains unclear how these mechanisms are modulated.

In summary, our results indicate that TM complex may be more effective than a single agent in the propelling test or writhing test. These effects are more evident in a gastrointestinal disorder model induced by WIR. This study might provide evidence that the combined treatment with trimebutine plus mosapride may produce a synergic effect on gastrointestinal motility and visceral pain control in gastrointestinal disorders. It is postulated that the synergic effect may result from a potentiating effect of one agent acting through a different pharmacological mechanism than the other agent.

\section{ACKNOWLEDGEMENTS}

This work was supported by Samil Pharmaceutical Co. Ltd. (Seoul, South Korea).

\section{REFERENCES}

Boyle, G., Mounsey, A. and Crowell, K. (2009) Clinical inquiries: what is the role of prokinetic agents for constipation? J. Fam. Pract. 58, 220d-220f.

Chicella, M. F., Batres, L. A., Heesters, M. S. and Dice, J. E. (2005) Prokinetic drug therapy in children: a review of current options. Ann. Pharmacother. 39, 706-711.

Chua, A. S., Bekkering, M., Rovati, L. C. and Keeling, P. W. (1994) Clinical efficacy and prokinetic effect of the CCK-A antagonist loxiglumide in nonulcer dyspepsia. Ann. N. Y. Acad. Sci. 713, 451-453.

Curran, M. P. and Robinson, D. M. (2008) Mosapride in gastrointestinal disorders. Drugs 68, 981-991.

Delco, M. L., Nieto, J. E., Craigmill, A. L., Stanley, S. D. and Snyder, J. R. (2007) Pharmacokinetics and in vitro effects of tegaserod, a serotonin 5-hydroxytryptamine $4(5-\mathrm{HT} 4)$ receptor agonist with prokinetic activity in horses. Vet. Ther. 8, 77-87.

Delvaux, M. and Wingate, D. (1997) Trimebutine: mechanism of action, effects on gastrointestinal function and clinical results. J. Int. Med. Res. 25, 225-246.

Dubinsky, B., Gebre-Mariam, S., Capetola, R. J. and Rosenthale, M. E. (1987) The antialgesic drugs: human therapeutic correlates of their potency in laboratory animal models of hyperalgesia. Agents Actions 20, 50-60.

Greenwood, B. and Davison, J. S. (1987) The relationship between gastrointestinal motility and secretion. Am. J. Physiol. 252, G1-7.

Hawkyard, C. V. and Koerner, R. J. (2007) The use of erythromycin as a gastrointestinal prokinetic agent in adult critical care: benefits versus risks. J. Antimicrob. Chemother. 59, 347-358.

Holzer, P. (2004) Opioids and opioid receptors in the enteric nervous system: from a problem in opioid analgesia to a possible new prokinetic therapy in humans. Neurosci. Lett. 361, 192-195.

Kii, Y., Nakatsuji, K., Nose, I., Yabuuchi, M., Mizuki, Y. and Ito, T. (2001) Effects of 5-HT(4) receptor agonists, cisapride and mosapride citrate on electrocardiogram in anaesthetized rats and guinea-pigs and conscious cats. Pharmacol. Toxicol. 89, 96-103.

Koop, H., Monnikes, H., Koop, I., Dionysius, J., Schwarz, C. and Arnold, R. (1986) Effect of the prokinetic drug cisapride on gastrointestinal hormone release. Scand. J. Gastroenterol. 21, 907-913.

Kountouras, J., Chatzopoulos, D., Zavos, C., Boura, P., Venizelos, J. and Kalis, A. (2002) Efficacy of trimebutine therapy in patients with gastroesophageal reflux disease and irritable bowel syndrome. Hepatogastroenterology 49, 193-197.
Luttecke, K. (1978) A trial of trimebutine in spastic colon. J. Int. Med. Res. 6, 86-88.

Matsue, T., Yamato, S. and Umeda, N. (1990) Mechanism of action of trimebutine maleate on gastro-colic reflex in patients with irritable bowel syndrome. Nippon Heikatsukin Gakkai Zasshi 26, 89-92.

Mine, Y., Yoshikawa, T., Oku, S., Nagai, R., Yoshida, N. and Hosoki, K. (1997) Comparison of effect of mosapride citrate and existing 5 -HT4 receptor agonists on gastrointestinal motility in vivo and in vitro. J. Pharmacol. Exp. Ther. 283, 1000-1008.

Nosaka, K., Takenaga, H., Magaribuchi, T. and Tamaki, H. (1984) Effects of trimebutine maleate (TM-906) on the gastrointestinal motility in anesthetized dogs. Nippon Heikatsukin Gakkai Zasshi 20, 407-412.

Odaka, T., Suzuki, T., Seza, A., Yamaguchi, T. and Saisho, H. (2006) Serotonin 5- HT4 receptor agonist (mosapride citrate). Nippon Rinsho 64, 1491-1494.

Reynolds, J. C. and Putnam, P. E. (1992) Prokinetic agents. Gastroenterol. Clin. North Am. 21, 567-596.

Roman, F. J., Lanet, S., Hamon, J., Brunelle, G., Maurin, A., Champeroux, P., Richard, S., Alessandri, N. and Gola, M. (1999) Pharmacological properties of trimebutine and $\mathrm{N}$-monodesmethyltrimebutine. J. Pharmacol. Exp. Ther. 289, 1391-1397.

Sakurai-Yamashita, Y., Takada, K., Takemura, K., Yamashita, K., Enjoji, A., Kanematsu, T. and Taniyama, K. (1999) Ability of mosapride to bind to 5-HT4 receptor in the human stomach. Jpn. J. Pharmacol. 79, 493-496.

Shimada, T., Kurachi, Y., Terano, A., Hamada, E. and Sugimoto, T. (1990) Trimebutine maleate has inhibitory effects on the voltagedependent $\mathrm{Ca} 2+$ inward current and other membrane currents in intestinal smooth muscle cells. Gastroenterol. Jpn. 25, 175-179.

Takagi, K. and Okabe, S. (1968) The effects of drugs on the production and recovery processes of the stress ulcer. Jpn. J. Pharmacol. 18, 9-18.

Takenaga, H., Magaribuchi, T. and Tamaki, H. (1984a) Effects of trimebutine maleate (TM-906) on the smooth muscles of isolated guinea pig gallbladder. Jpn. J. Pharmacol. 35, 439-443.

Takenaga, H., Magaribuchi, T. and Tamaki, H. (1984b) Effects of trimebutine maleate (TM-906) on the spontaneous contraction of isolated guinea pig colon. Jpn. J. Pharmacol. 34, 177-181.

Takenaga, H., Magaribuchi, T. and Tamaki, H. (1986) Effects of trimebutine maleate (TM-906) on the spontaneous contraction of isolated duodenum and ileum in both guinea pigs and rabbits. Jpn. J. Pharmacol. 40, 13-20.

Tanaka, M. (1998) Gastric ulcer, motility, and trimebutine. J. Gastroenterol. 33, 916-917.

Uchiyama, M., Iwafuchi, M., Yagi, M., linuma, Y., Kanada, S., Ohtaki, M. and Homma, S. (2000) Effects of trimebutine on intestinal motility after massive small bowel resection. J. Smooth. Muscle. Res. 36, $117-126$.

Washabau, R. J. (2003) Gastrointestinal motility disorders and gastrointestinal prokinetic therapy. Vet. Clin. North Am. Small Anim. Pract. 33, 1007-1028, vi.

Wingate, D., Hongo, M., Kellow, J., Lindberg, G. and Smout, A. (2002) Disorders of gastrointestinal motility: towards a new classification. J. Gastroenterol. Hepatol. 17(Suppl), S1-14.

Yoshida, N., Ito, T., Karasawa, T. and Itoh, Z. (1991) AS-4370, a new gastrokinetic agent, enhances upper gastrointestinal motor activity in conscious dogs. J. Pharmacol. Exp. Ther. 257, 781-787.

Yoshida, N., Omoya, H., Kato, S. and Ito, T. (1993) Pharmacological effects of the new gastroprokinetic agent mosapride citrate and its metabolites in experimental animals. Arzneimittelforschung 43, 1078-1083.

Zenilman, M. E. (1993) Origin and control of gastrointestinal motility. Surg. Clin. North. Am. 73, 1081-1099.

Zhang, H. Q., Zhou, C. H. and Wu, Y. Q. (2005) Effect of emodin on small intestinal peristalsis of mice and relevant mechanism. World J. Gastroenterol. 11, 3147-3150. 